\title{
Modeling aluminum using molecular dynamics simulation
}

\author{
H. Chabba ${ }^{1}$, M. Lemaalem ${ }^{2}$, A. Derouiche ${ }^{2}$, D. Dafir ${ }^{1}$ \\ ${ }^{1}$ Science and Engineering Technology, Laboratory for energy production and sustainable development, superior \\ school of technology Fez, P. O. Box 2427 Imouzzer road Fez,30000, Morocco. \\ ${ }^{2}$ Polymer Physics and Critical Phenomena Laboratory Sciences Faculty Ben M'sik \\ P. O. Box 7955, Casablanca, Morocco.
}

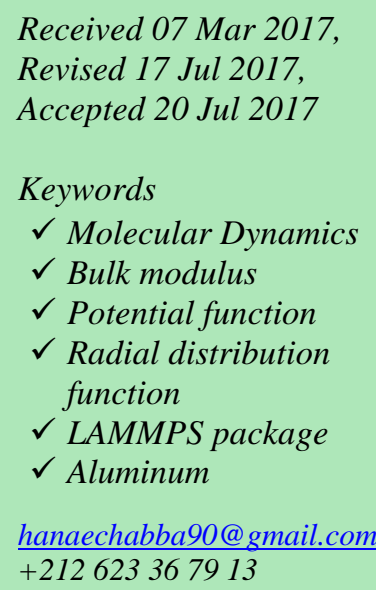

\begin{abstract}
The study of a material is done by the study of its mechanical, chemical, physical and thermal properties. The bulk modulus as one of the important mechanical properties of metals; the radial distribution function; describes how the density varies as a function of the distance to a particle of reference; are calculated using molecular dynamics simulation method. The box of simulation contains 4000 atoms of pure aluminum and two different potentials functions are utilized to predict the bulk modulus for pure aluminum. We have also calculated the radial distribution function, and the rupture pressure Therefore, all simulations were performed with the use of the code of molecular dynamics LAMMPS. We found that the potential functions to two body can predict the bulk modulus of aluminum in agreement with the experimental data.
\end{abstract}

\section{Introduction}

Molecular dynamics (MD) is simulation of physical movements of atoms and molecules. The atoms and molecules are allowed to interact for a period of time, giving a view of the motion of the atoms. The equations of motion are solved numerically the Newton's equations for a system of interacting particles to follow the time evolution of the system, allowing the derivation of kinetic and thermodynamic properties. There are several kinds of potential functions that are used in molecular dynamics simulation such as Lennard Jones (LJ), Embedded Atom Method (EAM), Modified Embedded Atom Method (MEAM), Tersoff which are used in different systems depending on the application. For variety of mechanical applications, the prediction of mechanical properties of aluminum is the question of great importance. Thousands of aluminum alloys with different chemical compositions and different microstructures are used in the industries while the most common methods to obtain their properties are time and money consuming experiments [1-2]. An important part of researches and development involve the innovation of new aluminum alloys [3]. Recently MD method has been extensively used for estimation of mechanical properties of aluminum alloys with different chemical compositions. In this paper, we have provided molecular dynamics simulation for calculating bulk modulus of pure aluminum and compared results of two different common potentials (LJ, EAM). We have found that EAM potential is more appropriate potential to predict the bulk modulus of pure aluminum and alloys.

Simulation permits the exploration of a wide range of properties, and it is able to address conditions which are difficult to be observing directly in experiment, this results in to providing a valuable complement to practical investigation.

In this contest, we use LAMMPS software Large-scale Atomic / Molecular Massively Parallel Simulator http://lammps.sandia.gov/index.html(Sandia National Laboratories).

This paper is organized as follows. In the first part, we give a brief recap of the bulk modulus and a description of pure aluminum; in the second part, we present and we conclude with results and discussion. 


\section{Materials and methods}

2.1. Aluminum

In general materials and metals in particular hold an important place in many industrial development or technological progress [3]. Aluminum is the second widely used metal in different fields like industry; due to its desirable chemical, physical and mechanical properties and it represents an important category of technological materials. The following table shows the aluminum characteristics in the ambient temperature:

Table 1: Identity card of pure aluminum [4].

\begin{tabular}{|l|l|}
\hline Aluminum Identity Card & $\mathrm{Al}$ \\
\hline Symbol & 13 \\
\hline Atomic number & $27 \mathrm{~g} /$ mole \\
\hline Atomic mass & $\mathrm{FCC} \mathrm{Structure}$ \\
\hline Structure & $660^{\circ} \mathrm{C}$ \\
\hline Melting temperature & $2056^{\circ} \mathrm{C}$ \\
\hline Boiling temperature & $68 \mathrm{GPa}$ \\
\hline Bulk modulus & $0.404 \mathrm{~nm}$ \\
\hline Lattice parameter & $69 \mathrm{GPa}$ \\
\hline Young's modulus & \\
\hline
\end{tabular}

These properties mean that the aluminum is used extensively in its pure state or more precisely in its unalloyed state (in most developed countries more than $40 \%$ of aluminum consumption takes place in the pure state) [5].

\subsection{Molecular dynamics}

Molecular dynamics (MD) is the term used to describe the solution of the classical equations of motion (Newton's equations) for a set of molecules [6-7].

The motion of molecules is generally governed by Newton's equations of motion in classical theory. In MD simulations, particle motion is simulated on a computer according to the equations of motion. Molecular dynamics simulation provides the methodology for detailed microscopic modeling on the molecular scale [8].

Classical Molecular dynamics is a computer simulation technique where the time evolution of a set of interacting atoms and molecules of a system is followed by integrating their equations of motion. The atoms and molecules of the system are allowed to interact for a period of time giving a view of their motion. The trajectories of the interacting particles are determined by numerically solving Newton's equations of motion where forces between the particles and potential energy are defined by molecular mechanic force field.

For each atom $\mathrm{i}$ of mass $\mathrm{m}$, we define the force $\mathrm{F}$ exerted by the whole system on this atom: [9].

$$
\begin{gathered}
F_{i}=m \frac{d^{2} r_{i}}{d t^{2}}=m \times a_{i} \\
F_{i}=-\nabla_{i} V=\frac{-d V}{d r_{i}}
\end{gathered}
$$

For this purpose, we need to be able to calculate the forces $F_{i}$ acting on the atoms, and these are usually derived from a potential energy $V\left(r^{N}\right)$ where $r^{N}=\left(r_{1} ; r_{2} ; \ldots ; r_{N}\right)$ represents the complete set of $3 \mathrm{~N}$ atomic coordinates.

\subsection{Potential of Lennard-Jones}

The potential of Lennard-Jones is a potential energy which serves to describe the interaction between two neutral atoms or molecules.

The empirical LJ function $V^{L J}$ describes the energy of dispersion interaction between atoms $\mathrm{i}$ and $\mathrm{j}$ that are separated by the distance $r_{i j}$, so $V^{L J}$ has the following form:

$$
V^{L J}\left(\vec{r}_{1}, \ldots, \vec{r}_{N}\right)=4 \epsilon\left(\left(\frac{\sigma}{r_{i j}}\right)^{12}-\left(\frac{\sigma}{r_{i j}}\right)^{6}\right)
$$

According to the above form, $\varepsilon$ is the depth of the potential and $\sigma$ is the distance at which the interaction energy between particles is zero [10]. 


\subsection{EAM potential}

The potentialEmbedded Atom Method (EAM)is widely used in molecular dynamics simulations for modeling metals and alloys; itis an approximation that describes the energy between atoms. The EAM was developed by Daw and Baskes [11]. Moreover, the energy is a function of a sum of functions of the separation between an atom and its neighbors. EAM is related to the second moment approximation to tight binding theory; these models are particularly appropriate for metallic systems.

The latter functions represent the electron density. The total energy of the system as two additive terms are pairwise sum of interactions between atoms and a term representing the electron density of each atomic site as shown in the equation below:

$$
\begin{gathered}
E_{i}=\sum_{i} F_{i}\left(\rho_{h, i}\right)+\frac{1}{2} \sum_{i} \sum_{j \neq i} \Phi_{i j}\left(r_{i j}\right) \\
\rho_{h, i}=\sum_{i \neq j} \rho_{j}\left(r_{i j}\right)
\end{gathered}
$$

The total energy in potential of the EAM $E_{i}$ consists of two parts, a pair potential $\Phi_{i j}\left(r_{i j}\right)$, and $F_{i}\left(\rho_{h, i}\right)$; the latter is the embedded function for atom $\mathrm{i}$ which depends on the electron density $\rho_{h, i}$ expressed by that atom $\mathrm{i}$ and $\mathrm{j}$ indicate the unique pairs of atoms within the $\mathrm{N}$ atoms of the system, $r_{i j}$ is their interatomic separation and $\rho_{j}\left(r_{i j}\right)$ is the density function [12].

\section{Characters}

\subsection{Bulk modulus}

The Bulk modulus of a substance is a measure of the compressibility of this substance. An object will be compressed in all directions when it is under pressure on all surfaces, so this pressure reduces the volume of a material. To compress liquids and solids, you must actually compress their atoms and molecules; since a compression of the entire object is equivalent to compressing each of its three dimensions. Therefore, the bulk modulus $B$ is a measure of the ability of a substance to withstand changes in volume while under compression in all sides. The change in volume is proportional to the force per unit area and its original volume, and it is related to the compressibility of the substance. Bulk modulus is the ratio of the pressure variation to the relative variation of the volume.

The relationship of the Bulk modulus to other physical quantities is explained in the expression below: [13].

Where:

$$
\begin{gathered}
B=-\Delta P \cdot \frac{\Delta V}{V_{0}} \\
\Delta V=V_{0}-V
\end{gathered}
$$

$\Delta \mathrm{V}$ : is standard deviation of instantaneous volume.

$\triangle \mathrm{P}$ : Pressure difference in GPa

B: Bulk modulus in GPa

$\Delta \mathrm{V}$ : Volume change

$\mathrm{V}$ : Volume after compression

$V_{0}$ : Initial volume

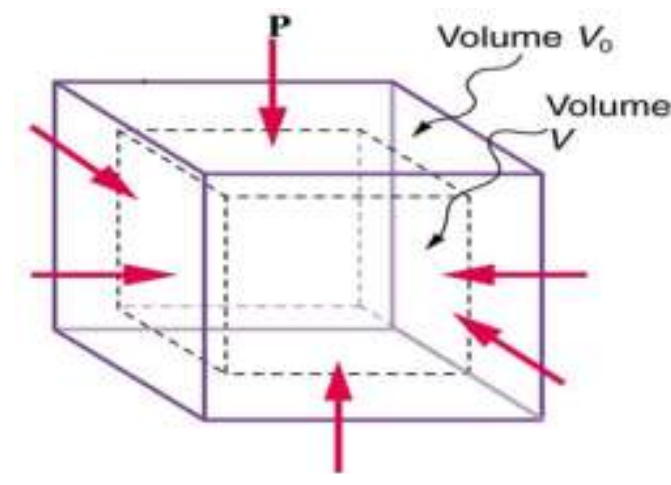

Figure 1: Schematic describe the bulk modulus

\subsection{Radial distribution function $R D F$ :}

The radial distribution function (Pair correlation function) $g(r)$ in a particularly system (atoms, molecules, colloids, etc.), is the most important structural quantities characterizing a system [14];describes how the density varies as a function of the distance to a particle of reference.

If a given particle is considered to be the origin $O$ and if $\rho$ is the average density in number of particles, then the density local average as a function of time at a distance $r$ from $O$ is $G(r)$. It is a measure of the probability of finding a particle at a distance of the distance a particle is given in reference, in comparison to that of an ideal gas. 
The radial distribution function provides information on the radial distribution of a type element around a component considered.

The functions of the radial distributions FDR are evaluated by the following equation [15-16]:

$$
g(r)=4 \pi r^{2} d r
$$

\section{Computer Packages:}

1.3. Background LAMMPS

LAMMPS is a classical molecular dynamics code. It was developed at Sandia National Laboratories, which is under United States Department of energy. It is distributed in the form of open source code under the GNU General Public License.

\subsection{Visualization of the system}

We have used the program Visual Molecular Dynamics (VMD) for display of molecular assemblies and viewing the systems from the simulations. It gives a simple representation of atoms and of the connections http://www.ovito.org/.

\section{Results and discussion}

\subsection{Structure}

In this research, we carried out molecular dynamics simulation deformation studies of Aluminum by traction. The effect of composition and strain rate were investigated. The following paragraphs describe the methods of creation of crystalline alloys and their analysis by radial distribution function followed by indentation studies of the alloys.

The molecular dynamics simulations were conducted with the software LAMMPS which has been adapted to calculate the bulk modulus. The traction applied to the pure aluminum and estimated the radial distribution function; we used two potentials (EAM and LJ).

In the first part, we have considered 4000 atoms of pure $\mathrm{Al}$, we used two types of potentials with periodic boundary conditions in a FCC lattice configuration. Then we start to run simulation with temperature of $1000 \mathrm{~K}$ at $100[\mathrm{kPa}]$ pressure, with time step of 10 femtoseconds and the simulation continuous for 0.001 time steps. We apply an artificial deformation force to a FCC Al, we used LAMMPS to calculate the Al FCC bulk modulus, and we measure stress/strain relationship and the radial distribution function.

The atomic structure of metals and their conditions of crystallization strongly influences the mechanical behavior resulting in the solid state. In the case of aluminum, the following figure describes atomic organization of pure aluminum to three dimensions before and after deformation.

The figure 2, shows a typical configuration from the simulation. In this case the aluminum system was described with VMD http://www.ovito.org/. We can conclude that, in this simple simulation, the microstructure changes after traction.

a

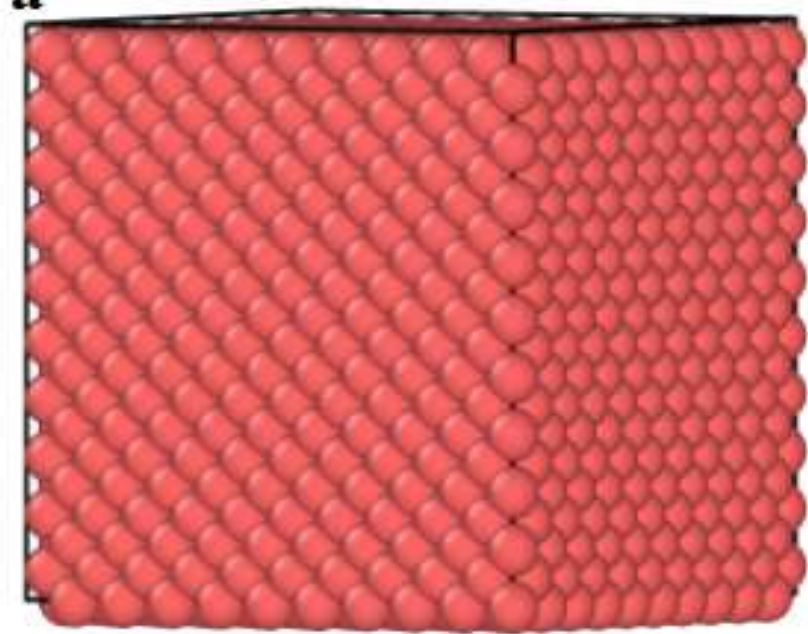

b

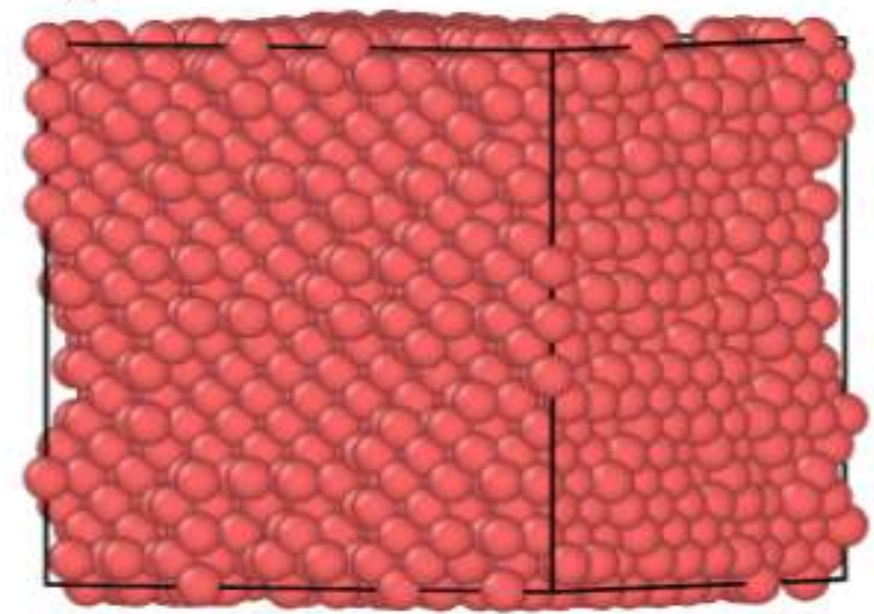

Figure 2: Atomic structure of pure aluminum: (a) represents the structure before the deformation, (b)after the deformation. 
After the deformation, the crystal structure of pure aluminum changed, the following table presents the percentages of each structure after deformation.

The atoms during deformation change their crystal structure. This effect is observed by the calculations made by the both potentials EAM and LJ.

Table 2: Percentages of each structure after deformation.

\begin{tabular}{|l|l|l|l|l|}
\hline Potentials & FCC & HCP & BCC & Others \\
\hline EAM potential & 74 & 2.3 & 0.4 & 23.3 \\
\hline LJ potential & 49.9 & 50 & 0.1 & 0 \\
\hline
\end{tabular}

\section{2. graph stress-strain}

The simulation is performed using molecular dynamics after constructing the model and stabilized it for the tensile test. This template allows us to predict the mode of deformation as well as the resulting rheology at the atomic scale.

The issue is to simulate the aluminum using two potentials to represent the graph of deformation.

In the case of pure aluminum, we obtain the graph of traction below. According to this graph, pure aluminum has only an elastic deformation, the linear part, and the non-linear part represents the rupture of the material, for the two potentials there is no elongation at rupture. During the traction, the deformation of Aluminum appears in the early stages.

The figure shows that atoms are distorted from the beginning of the test and begin to deform locally, this phenomenon is reproduced for the two types of potential.

The constraint is evolving more regularly in function of the strain, as observed on the graph we can conclude that in pure aluminum plastic deformation appears very early and gradually. From which the crystalline phase begins to be distorted. This decrease is the result of the destruction of the material.

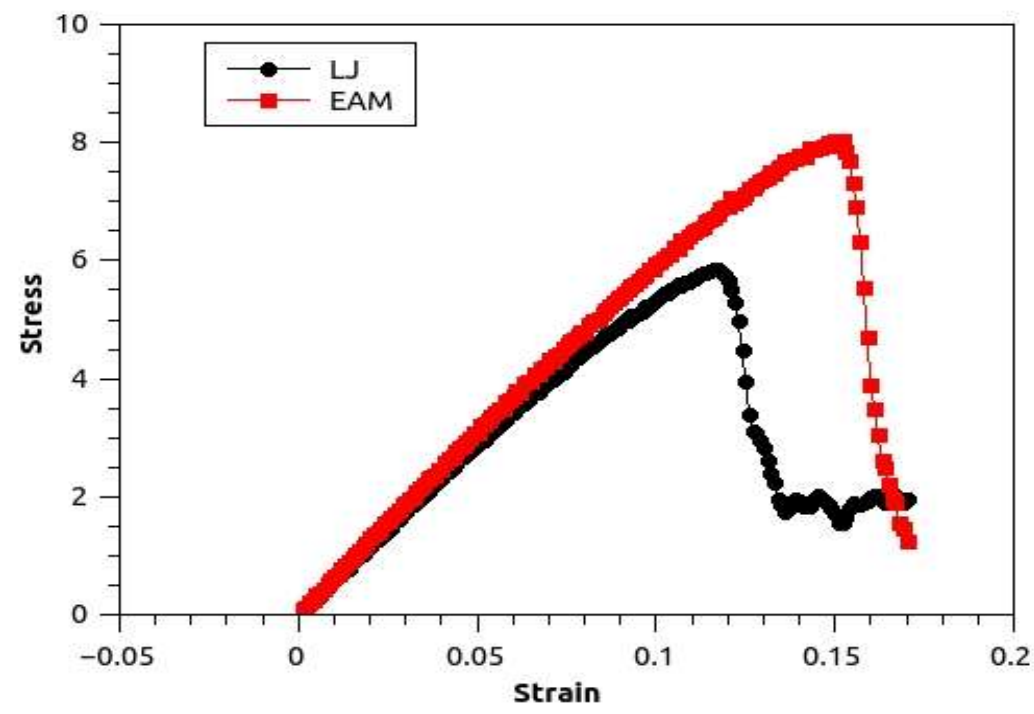

Figure 3: Stress-strain graph established according to the measurements made during the deformation

As shown in the graph, when we change the potential we have had a change of the bulk modulus from change of the module of Hooke and the pressure of rupture that was obtained from the potential LJ. The latter is different to the one that was obtained by the potential EAM.

\subsection{Bulk modulus}

It determines the strength of rupture of a tube of the alloy by a tensile test, the values of the bulk modulus and the pressure of rupture for pure aluminum are given in Table 3.

The potential depends on the pressure applied, therefore, when we changed the potential, the force applied on the material changes the pressure of rupture.

As shown in Table 3, the bulk modulus is in good agreements with experimental data, taking into account both types of potentials, [17]. So, as we expected the LJ and the EAM potential are appropriate for predicting the bulk modulus of Aluminum. This result means that simulations are able to describe the behavior of aluminum. 
Table 3: The bulk modulus and the pressure of rupture for pure aluminum

\begin{tabular}{|l|l|l|}
\cline { 2 - 3 } \multicolumn{1}{c|}{} & Bulk modulus (GPa) & Rupture pressure \\
\hline LJ potential & 58 & 5.85 \\
\hline EAM potential & 59 & 8.03 \\
\hline Experimental value & 68 & \\
\hline
\end{tabular}

\subsection{Radial distribution function $R D F$}

Radial distribution function is measure of arrangement of atoms in material.

The atomic RDF $\mathrm{g}(\mathrm{r})$ shows peaks corresponding to the inter-atomic distances existing in the material. It can provide information for both the average structure and the local atomic arrangement. It allows for a direct analysis of the inter-atomic distance and atomic displacements without prior knowledge of the structure. It also gives a measure of crystalline nature of a material. So, more is the close packing, more peaks of higher values will be observed at lower radial distances.

In the second part, we use EAM and LJ potential function to simulate and calculate Radial Distribution Function $\mathrm{Al}-\mathrm{Al}$ in function of the distance of a system of 4000 atoms. These functions correspond to the radial density of the atoms of type $\beta$ around the atoms of type $\alpha$. The results are presented in figure 4 .

As shown in Figure 4, It can be noted that the FDRs Al-Al of the system obtained by the potential EAM is different to the one obtained by the potential $\mathrm{LJ}$; it is observed that there is of the oscillations after the first peak of the two graphs indicating the existence of a sequence more marked. The first peaks are higher and finer, which characterizes a more interesting local order. And as the peak is different on the two graphs, therefore, we find that the distance $\mathrm{Al}-\mathrm{Al}$ is in function of the potential. For the potential EAM, the first peak appears at a distance of $3 \AA$, but for the potential LJ the oscillations have appeared at to $2.4 \AA$ of the simulated aluminum.

The peak means that it is the greatest probability of distance $\mathrm{Al}-\mathrm{Al}$ and this result is in agreement with the experimental results for Al-Al [18]. This distance binds the more frequently of the two atoms, and the first maximum of the radial distribution functions corresponds to the length of the interatomic connection the more probably.

Except for the systems $\mathrm{Al}-\mathrm{Al}$ of 4000 atoms, the main differences between the two types of potential: the position of the maxima of the FDRs calculated is $4.3 \AA$ by the use of EAM and $6 \AA$ by the use of the potential LJ.

The figure shows the FDRs Al-Al of the system of 4000 atoms; the first maximum of the FDRs corresponds to the length of the interatomic connection the more probably. The FDRs is obtained by the potential EAM and LJ. In the sense that, the position of the maxima of the FDRs Al-Al is respectively 4.3 and 6.

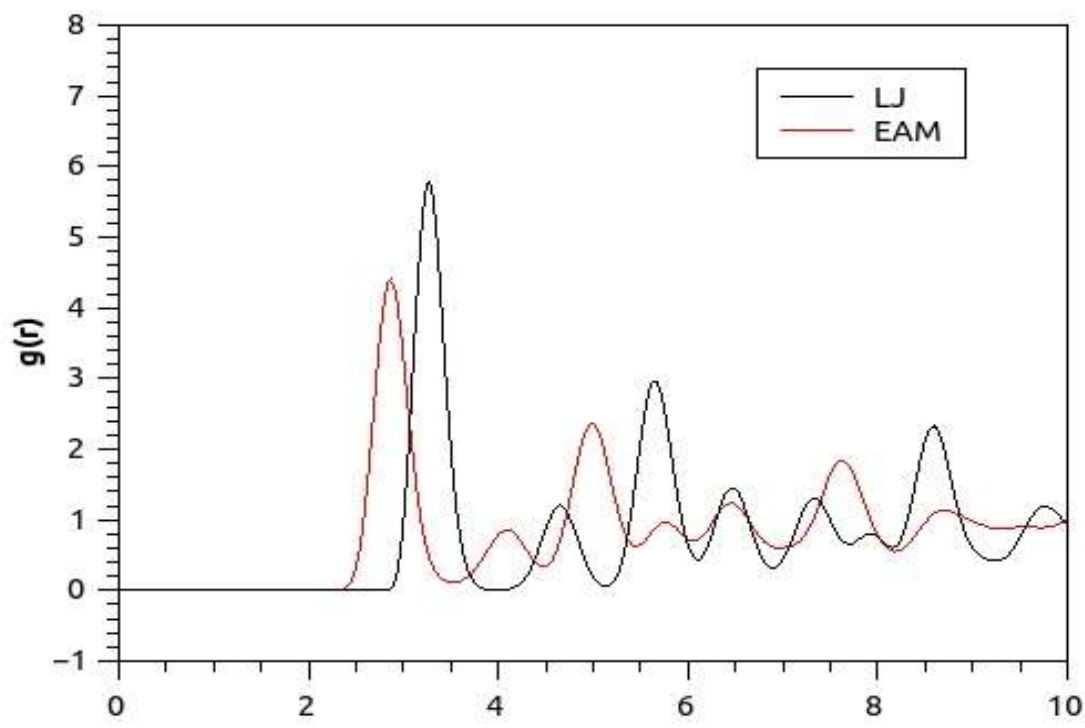

Figure 4: Radial distribution function of pure aluminum in function of the distance between two atoms calculated in molecular dynamics by EAM potential (red) and Leonard-Jones (black).

Moreover, the results obtained are compared with experimental measurements from literature. Looking at table 4 , we see that EAM potential is slightly more accurate than the LJ potential, for pure aluminum. 
Table 4: Distances interatomic obtained from the FDRs of the system Al-Al.

\begin{tabular}{|l|l|}
\cline { 2 - 2 } \multicolumn{1}{l|}{} & Distance interatomic $(\mathbf{\AA})$ \\
\hline LJ potential & 2.4 \\
\hline EAM & 3 \\
\hline Experimental values & 3.24 \\
\hline
\end{tabular}

\section{Conclusions}

In summary, in order to better understand the deformation mechanism, Molecular Dynamics simulation was performed with two types of potentials, for $\mathrm{Al}$ during deformation.

The modeling of the traction of pure aluminum by Molecular Dynamics allows us to predict the deformation mechanisms. It allows also, to conclude on details microstructural hardly accessible by other methods and to make the link with mechanical behavior microscopic in a small deformation.

As conclusion, it can be stated according to results obtained and compared with actual measurements, that the potential EAM and LJ potential are appropriate potential functions for predicting the bulk modulus, radial distribution function Al-Al, Interatomic distance and the rupture pressure of pure aluminum. It is also apparent from this study that the results obtained by EAM potential is more accurate than those obtained by LJ Potential.

\section{References}

1. S.C. Sharma, B. Girish, R. Kamath, and B.M. Sathish, J. Mater. Eng. Perform. 8(3), (1999), 309-314.

2. K.H.W. Seah, S.C. Sharma, and M. Krishna, J. ASTM Int., 3(3), (2006), 1-5.

3. L. F. Mondolfo, Aluminum Alloys Structures and Properties, Butterworth, London, 12, 0-40870680-5, (1976), 1-3.

4. J. Randolph Kissell, L. Robert Ferry, Aluminum structures a guide to their specifications and design second edition, John wiley \& sons, New York, 0-471-01965-8, (1996), 18.

5. J. Idrac, Thesis of Doctor, Institute Nation Polytechnique of Toulouse, (2005).

6. M. P. Allen \& D. J. Tildesley, Computer Simulation of liquids, Oxford University press, New York, 019 855645, (1987), 17.

7. P. Dea, T. Frauenheim, M. R. Pederson (eds.), Computer Simulation of Materials at Atomic Level, ISBN: 3-527-40290-X, Volume 1, 2000.

8. D. C. Rapaport, The Art of Molecular Dynamics Simulation second edition, Cambridge University Press, 13 978-0-511-19448-1, (2004), 11-14.

9. D. Frenkel, B. Smith, Understanding molecular dynamics simulation from algorithms to applications, second edition, Academic Press, London, 0-12-267351-4 (1996) 63-67.

10. P. Guan, D. R. Mckenzie, B. A. Pailthorpe, J. Condens. Matter Phys., 8, 875, (1996).

11. M.S. Daw, M. I. Baskes, Phys. Rev. B, 29, (1983), 6443.

12. S. M. Foiles, M. I. Baskes, M. S. Daw, Phys. Rev. B, 33, (1986), 7983-7991.

13. A. Rajabpour, L. Seidabadi, M. Soltanpour, Procedia Mater. Sci., 11, (2015), 391-396.

14. H. Akbarzadeh, M. Abbaspour, J. Mol. Liq. 219 (2016) 158-164.

15. B. Widom, Statistical mechanics a concise introduction for chemists, Cambridge University Press, New York, 052181119 8, (2002) 88-98.

16. D. Cheng, J. Lan, Mol. Simul. 36 (2010) 805.

17. W.C. Oliver, G.M. Pharr, J. Mater. Res., 7, (1992), 6.

18. P. Ganster, Doctoral Thesis, University Montpellier II Sciences and Techniques of Languedoc, (2004).

(2018) ; http://www.jmaterenvironsci.com 